\title{
PURIFICAÇÃO E CARACTERIZAÇÃO DA $\beta$-LAPACHONA E ESTUDO DE ESTABILIDADE DOS CRISTAIS EM DIFERENTES CONDIÇÕES DE ARMAZENAMENTO
}

\author{
Geisiane Maria Cavalcante Alves, Larissa Araújo Rolim, Pedro José Rolim Neto*, Ana Cristina Lima Leite e Dalci \\ José Brondani \\ Departamento de Ciências Farmacêuticas, Universidade Federal de Pernambuco, Rua Prof. Arthur de Sá, s/n, Cidade Universitária,
} 50740-52 Recife - PE, Brasil

Flávia Patrícia Morais de Medeiros

Laboratório Farmacêutico do Estado de Pernambuco, Largo de Dois Irmãos, 1117, 52171-010 Recife - PE, Brasil

Lothar W. Bieber

Departamento de Química Fundamental, Universidade Federal de Pernambuco, Av. Prof. Luiz Freire, s/n, Cidade Universitária, 50740-540 Recife - PE, Brasil

Francisco Jaime Bezerra Mendonça Junior

Departamento de Antibióticos, Universidade Federal de Pernambuco, Rua Prof. Arthur de Sá, s/n, Cidade Universitária, 50740-52 Recife - PE, Brasil

Recebido em 3/1/07; aceito em 21/6/07; publicado na web em 19/12/07

\begin{abstract}
PURIFICATION AND CHARACTERIZATION OF $\beta$-LAPACHONE AND STABILITY STUDY OF THE CRYSTALS UNDER DIFFERENT STORING CONDITIONS. The $\beta$-lapachone is a product obtained from the Ipê Roxo tree [Tabebuia avellandae], which has proved its excellent antineoplasic potential acting through a particular mechanism of apoptosis against various cancer types. This study aims at determining the identity card of $\beta$-lapachone by means of physico-chemical and pharmaco-technical characterization. A purifying process has been performed, as well as the isolation of a contaminant, its isomer $\alpha$-lapachone. A stability study was also performed, determining the ideal storing conditions for $\beta$-lapachone, essential for the ongoing pre-formulation studies for obtaining the different classic pharmaceutical forms and modified release systems.
\end{abstract}

Keywords: $\beta$-lapachone; characterization and stability study.

\section{INTRODUÇÃO}

A $\beta$-lapachona $\left(\mathrm{C}_{15} \mathrm{H}_{14} \mathrm{O}_{3}, \mathrm{MM} 242,3\right)$, conhecida quimicamente por 3,4-diidro-2,2-dimetil-2H-naftol[1,2-b]pirano-5,6-diona, é uma ortonaftoquinona de ocorrência natural isolada do ipê-roxo, ou paud'arco roxo (Tabebuia avellandae Lor), da família Bignoneaceae, que cresce principalmente no Brasil ${ }^{1-4}$. É um produto vegetal simples que tem demonstrado excelente potencial antineoplásico, agindo por um mecanismo particular de apoptose contra diversos tipos de câncer, em especial algumas linhagens de próstata refratárias aos tratamentos convencionais ${ }^{5-8}$. Está sendo desenvolvido pelo Laboratório Farmacêutico do Estado de Pernambuco - LAFEPE, como parte integrante de seu elenco oncológico, um medicamento à base de $\beta$-lapachona na forma farmacêutica cápsula gelatinosa mole.

Para isso, é de suma importância a caracterização físico-química do princípio ativo pois, dessa forma, se estabelece a carta de identidade do produto, possibilitando sua padronização e avaliação de sua pureza, tornando-o adequado para a realização de estudos de pré-formulação.

Este trabalho relata métodos específicos para a determinação de pureza da $\beta$-lapachona e sua caracterização físico-química e farmacotécnica, por diversas metodologias.

\section{MÉTODOS EXPERIMENTAIS}

\section{Equipamentos}

Na purificação dos lotes 402 e 702 de $\beta$-lapachona foram utili-

*e-mail: pedro.rolim@pq.cnpq.br zados uma estufa de secagem Fanem ${ }^{\circledR}$ modelo $315 / 3$ e luz ultravioleta Spectroline ${ }^{\circledast}$ modelo ENF-240C para revelação das placas cromatográficas de camada delgada.

Para os testes analíticos realizados na caracterização da $\beta$ lapachona lote 103, utilizou-se os seguintes equipamentos: análises microscópicas - microscópio óptico Zeizz Axiolab ${ }^{\circledR}$ com câmara acoplada MC 80; ponto de fusão - fusiômetro Polax ${ }^{\oplus}$ WRS1; umidade por perda por incineração - balança Metter Toledo ${ }^{\circledR}$ HB43 Hologen Moisture Analyzer, e por Karl Fischer - Mettler ${ }^{\circledR}$ DL-18; análise granulométrica - microscópio óptico Lieka - Leitz Bimed $^{\circledR}$ com objetiva de 40x.

O sistema cromatográfico utilizado para o doseamento consistiu num cromatógrafo líquido de alta eficiência HP (HewlettPackard $^{\circledR}$ ) Série 1100 equipado com sistema de bombas quaternário, degaseificador por ultra-som, detector UV-VIS e injetor automático, acoplado a um integrador de dados HP 3395 (Hewlett-Packard ${ }^{\circledR}$ ). A coluna utilizada foi uma Shim-pack 100 CLC(M) ODS $\left(\mathrm{C}_{18}\right)$ de fase reversa, com $46 \mathrm{~mm} \times 25 \mathrm{~cm}$ e partículas esféricas de $5 \mu \mathrm{m}$ de diâmetro $\left(\right.$ Shimadzu $\left.{ }^{\circledR}\right)$.

As curvas de calorimetria exploratória diferencial (DSC convencional) foram obtidas num calorímetro Shimadzu ${ }^{\circledR}$ modelo DSC50 interligado aos softwares Shimadzu ${ }^{\circledR}$ TA-60WS/TA-50WS. O sistema de DSC-fotovisual foi constituído pelo mesmo equipamento utilizado no DSC convencional acoplado a um microscópio Olympus $^{\circledR}$ conectado a uma câmara fotográfica Sanyo $^{\circledR}$ de alta resolução modelo VCC-D520, com imagem interligada ao software Intel Smart ${ }^{\circledR}$ Vídeo Record II. As curvas de termogravimetria (TG) foram obtidas numa termobalança Shimadzu ${ }^{\circledR}$ modelo TGA-50H.

Os espectros de infravermelho (IVTF) foram realizados em 
Espectrofotômetro de IVTF Bruker ${ }^{\circledR}$ modelo IFS66 (Fourier TranformFT) utilizando pastilhas de $\mathrm{KBr}$. O espectro de varredura no ultravioleta foi obtido em espectrofotometro Shimadzu ${ }^{\circledR}$ UV-VIS 2401 PC. As ressonâncias magnéticas nucleares protônicas $\left(\mathrm{RMN}^{1} \mathrm{H}\right)$ e de carbono $13\left(\mathrm{RMN}^{13} \mathrm{C}\right)$ foram realizadas em espectrômetro Varian ${ }^{\circledR}$, modelo Unity plus-300 MHz, utilizando clorofórmio deuterado (Tedia ${ }^{\circledR}$ ) como solvente. Para a espectrometria de massa (EM) foi utilizado um espectrômetro Finnigan GCQ Mat tipo quadrupolo íon-trap, acoplado a um cromatógrafo a gás $\mathrm{HP}^{\circledR} 5890$ Series II.

Para o estudo de estabilidade dos cristais foi utilizada uma câmara climática Fanem ${ }^{\circledR}$ modelo 345, dessecador Satelit ${ }^{\circledR}$ HS 320 e refrigerador Electrolux ${ }^{\circledR}$ modelo R130.

\section{Materiais}

Foram utilizados dois lotes de $\beta$-lapachona matéria-prima, produzidos pelo Departamento de Antibióticos da Universidade Federal de Pernambuco (UFPE), L. 402 e L. 702, e um lote obtido através da purificação dos dois lotes anteriores, L. 103. Foi utilizado também um padrão secundário de $\beta$-lapachona produzido pela UFPE, padronizado a partir do padrão primário Sigma ${ }^{\circledR}$ Lote $31 \mathrm{~K} 1558$.

$\mathrm{Na}$ etapa de purificação foram utilizados etanol P.A., acetato de etila e tolueno $\left(\right.$ Merck $^{\circledR}$ ) e placas cromatográficas pré-fabricadas com camada adsorvente de dióxido de silício poroso sintético e sílica gel $60 \mathrm{~F}_{254}$, depositada em uma lâmina de alumínio $\left(\operatorname{Merck}^{\circledR}\right)$.

Acetonitrila, grau HPLC (Tedia ${ }^{\circledR}$ ), água ultrapurificada pelo sistema Milli-Q ${ }^{\circledR}$ Plus (Millipore ${ }^{\circledR}$ ) e ácido acético glacial $\left(\right.$ Merck $^{\circledR}$ ) foram utilizados para preparação da fase móvel, amostras e curva controle na realização dos doseamentos.

Membranas tipo HV com $47 \mathrm{~mm}$ de diâmetro e $0,45 \mu \mathrm{m}$ de diâmetro do poro $\left(\right.$ Supelco $\left.^{\circledR}\right)$ e unidades filtrantes Millex ${ }^{\mathrm{TM}}$ tipo HV com $13 \mathrm{~mm}$ de diâmetro e $0,45 \mu \mathrm{m}$ de diâmetro do poro (Millipore $^{\circledast}$ ), foram utilizadas para filtração da fase móvel e das amostras, respectivamente. Papel de filtro quantitativo com 125,0 $\pm 1,0 \mathrm{~mm}$ também foi utilizado no preparo das amostras (Framex ${ }^{\circledR}$ ).

\section{Processo de purificação}

Os dois lotes de $\beta$-lapachona contaminados, L. 402 e L. 702, foram utilizados para obtenção de um terceiro lote puro, L. 103. Os lotes contaminados foram solubilizados a quente no solvente utilizado no processo de semi-síntese da $\beta$-lapachona, etanol PA. Após recristalização, os cristais formados foram filtrados a vácuo e colocados em estufa até completa secagem a $80{ }^{\circ} \mathrm{C}$. A pureza foi verificada através da análise de cromatografia em camada delgada (CCD). A fase móvel utilizada foi composta de tolueno:acetato de etila (9:1). A revelação das placas foi realizada através de luz ultravioleta a $254 \mathrm{~nm}$.

O contaminante foi isolado por cromatografia em coluna de sílica gel, 70-230 mesh, $60 \AA$ (Aldrich Chemical ${ }^{\circledR}$ ) utilizando o mesmo sistema eluente da CCD e foi identificado por espectroscopia de ressonância magnética nuclear protônica $\left(\mathrm{RMN}^{1} \mathrm{H}\right)$ e de infravermelho (IVTF).

\section{Caracterização}

Toda a caracterização foi realizada com o lote 103 purificado, seguindo as especificações para cada método.

\section{Condições cromatográficas utilizadas para o doseamento}

O doseamento foi realizado por cromatografia líquida de alta eficiência (CLAE). A fase móvel foi obtida realizando uma mistu- ra de acetonitrila:ácido acético 0,25\% (50:50, v/v). A razão do fluxo da fase móvel foi de $2 \mathrm{~mL} / \mathrm{min}$, o volume de injeção foi de 20 $\mu \mathrm{L}$ utilizando um comprimento de onda fixado em $254 \mathrm{~nm}$. A coluna deve ser mantida a uma temperatura de $25{ }^{\circ} \mathrm{C}$. As amostras de $\beta$-lapachona foram preparadas em acetonitrila com posterior diluição em fase móvel obtendo uma concentração final de $5 \mu \mathrm{g} / \mathrm{mL}$.

Para cada corrida cromatográfica realizada, foi feita uma curva de calibração, para obtenção das concentrações das amostras do dia. Inicialmente foi preparada uma solução mãe de $\beta$-lapachona padrão secundário em acetonitrila obtendo-se uma concentração de $10 \mu \mathrm{g} / \mathrm{mL}$. Posteriores diluições em fase móvel foram feitas para obtenção das seguintes concentrações: 0,$125 ; 1$ e $8 \mu \mathrm{g} / \mathrm{mL}$.

\section{Estudo calorimétrico}

Antes de iniciar as análises de DSC e DSC-fotovisual o equipamento foi calibrado, em relação à temperatura, com os padrões índio $\left(156,6^{\circ} \mathrm{C} \pm 0,2\right)$ e zinco $\left(419,5^{\circ} \mathrm{C} \pm 0,3\right)$ através de seus pontos de fusão. Os fluxos de calor e entalpia foram calibrados com o calor de fusão do índio $(28,58 \mathrm{~J} / \mathrm{g} \pm 0,30)$ usando as mesmas condições das amostras. O fator de correção foi calculado de acordo com os procedimentos e especificações da Shimadzu ${ }^{\circledR}$.

A curva de DSC convencional foi obtida com uma atmosfera de nitrogênio de $50 \mathrm{~mL} \min ^{-1}$ e razão de aquecimento de $10{ }^{\circ} \mathrm{C} \mathrm{min}^{-1}$, numa faixa de temperatura de $25-400{ }^{\circ} \mathrm{C}$. As amostras foram colocadas em uma célula de alumínio fechada com massa de $2 \mathrm{mg}$ $\pm 0,03$. As fotos do DSC-fotovisual foram obtidas com razão de aquecimento de $10{ }^{\circ} \mathrm{C} \mathrm{min}^{-1}$, em uma faixa de temperatura variando entre $25-240{ }^{\circ} \mathrm{C}$, com célula de alumínio aberta.

A curva TG foi obtida em atmosfera de ar sintético com fluxo de $20 \mathrm{~mL} \min ^{-1}$, na razão de aquecimento de $10{ }^{\circ} \mathrm{C} \mathrm{min}^{-1}$, numa faixa de temperatura de $25-900{ }^{\circ} \mathrm{C}$, utilizando os softwares Shimadzu TA-60WS/TA-50WS. A amostra foi empacotada em uma célula de alumínio com massa de $5,12 \mathrm{mg}$.

\section{Caracterização farmacotécnica}

As propriedades de compactação da $\beta$-lapachona foram obtidas através da determinação da capacidade de compactação, densidade aparente $\left(\mathrm{d}_{\mathrm{AP}}\right)$ e densidade de compactação $\left(\mathrm{d}_{\mathrm{CP}}\right)$. Este ensaio foi realizado com $100 \mathrm{~g}$ de $\beta$-lapachona em um compactador automático (desenvolvimento local) provido de uma proveta normatizada. $\mathrm{O}$ equipamento promove uma compactação do pó através de movimentos verticais repetidos na proveta. $\mathrm{O}$ volume inicial $\left(\mathrm{V}_{0}\right)$ ocupado pelo produto foi medido, com posterior medição após 10 compactações, necessárias para acomodação do pó $\left(\mathrm{V}_{10}\right), 500$ compactações $\left(\mathrm{V}_{500}\right)$ e 1250 compactações $\left(\mathrm{V}_{1250}\right)$. Ao final das compactações foi possível a determinação da capacidade de compactação pela subtração de $\mathrm{V}_{10}-\mathrm{V}_{1250}$, onde valores acima de $20 \mathrm{~mL}$ são considerados inadequados para manipulação de formas sólidas, pois dificultariam o enchimento de câmaras ou de cápsulas. A relação entre a massa de $\beta$-lapachona e o volume ocupado pelo pó antes e após a compactação determinou as densidades aparente e de compactação, respectivamente?

O Índice de Carr exprime em forma percentual a capacidade de compactabilidade e compressibilidade de um pó ou granulado. É dado pela Equação 1, onde valores entre 5 e $15 \%$ são considerados com excelente fluxo ${ }^{10}$ :

$\mathrm{I}_{\mathrm{CARR}}(\%)=\left(\mathrm{d}_{\mathrm{CP}}-\mathrm{d}_{\mathrm{AP}}\right) * 100 / \mathrm{d}_{\mathrm{CP}}$

Um índice similar, de interpretação mais simples, foi proposto por Hausner (1967) (Equação 2), onde valores menores que 1,25 
indicam bom fluxo; valores maiores que 1,5 indicam fluxo ruim; valores entre 1,25 e 1,5 exigem a adição de lubrificantes para melhorar o escoamento ${ }^{10}$

$\mathrm{I}_{\text {HAUSNER }}=\mathrm{d}_{\mathrm{CP}} / \mathrm{d}_{\mathrm{AP}}$

Estes índices são determinações pontuais, exprimindo apenas o potencial de compactação/compressão, e não a facilidade ou velocidade com que estas ocorrem. Para este tipo de avaliação temos o ângulo de repouso e o tempo de escoamento ${ }^{10}$.

$\mathrm{O}$ ângulo de repouso é um parâmetro que tem como finalidade avaliar a dificuldade que um pó tem para fluir livremente através de um orifício para uma superfície livre. Deixando um pó fluir livremente através de um orifício sobre uma superfície plana será formado um cone, onde o ângulo da base desse cone é chamado de ângulo de repouso, ou seja, é a relação entre a altura (h) e o raio (r) do cone formado. Considera-se que um pó tem boas propriedades de escoamento quando possui ângulo de repouso igual ou inferior a $30^{\circ}$. Ângulos superiores a $40^{\circ}$ sugerem difícil fluxo ${ }^{9}$. Esse ângulo foi determinado pelo cone resultante do escoamento de $100 \mathrm{~g}$ de pó em um funil normatizado, cuja abertura de saída encontrava-se a $20 \mathrm{~cm}$ de altura, sobre uma folha de papel milimetrado. Foi calculado o valor médio de três determinações. Paralelamente ao ângulo de repouso, foi determinado o tempo de escoamento, onde resultados acima de $10 \mathrm{~s}$ são considerados com tempo de escoamento infinito.

\section{Estudo de estabilidade dos cristais}

Os cristais de $\beta$-lapachona foram colocados em 5 condições de armazenamento diferentes, com o propósito de monitorar as instabilidades ocorridas em cada condição: temperatura ambiente protegido da luz, temperatura ambiente exposto à luz fluorescente, dessecador, refrigerador com temperatura de $2 \pm 2{ }^{\circ} \mathrm{C}$ e câmara climática com temperatura de $40 \pm 2{ }^{\circ} \mathrm{C}$ e umidade relativa de $75 \pm$ $5 \%$, com o objetivo de aumentar a velocidade de degradação química e modificações físicas.

Os tempos de análises do estudo foram de 0, 30, 60, 90 e 180 dias e foram realizadas avaliações macroscópicas, microscópicas, por CCD e por CLAE, com os mesmos parâmetros utilizados anteriormente ${ }^{11}$.

\section{RESULTADOS E DISCUSSÃO}

\section{Processo de purificação}

$\mathrm{Na}$ análise de CCD da $\beta$-lapachona, Rf 0,25 , foi possível verificar a diferença de pureza entre as amostras antes e após o processo de purificação, através da observação da presença de contaminação pelo isômero $\alpha$-lapachona, com $\mathrm{Rf}$ de 0,50 , originado no processo de semi-síntese não padronizado da droga, que foi identificado por espectroscopia de RMN ${ }^{1} \mathrm{H}$ e de IVTF, estando de acordo com a literatura $^{12,13}$. Foram necessários três ciclos de recristalização para obtenção de uma matéria-prima livre de $\alpha$-lapachona, quando analisada por CCD.

\section{Caracterização}

A $\beta$-lapachona possui uma forma bem definida de cristais aciculares, laranja-avermelhado, visíveis a olho nu (Figura 1).

Os cristais observados no microscópio óptico para análise granulométrica tinham um tamanho praticamente uniforme, originando um tamanho médio de 233,1 $\mu \mathrm{m}$ como observado na Figura 2.

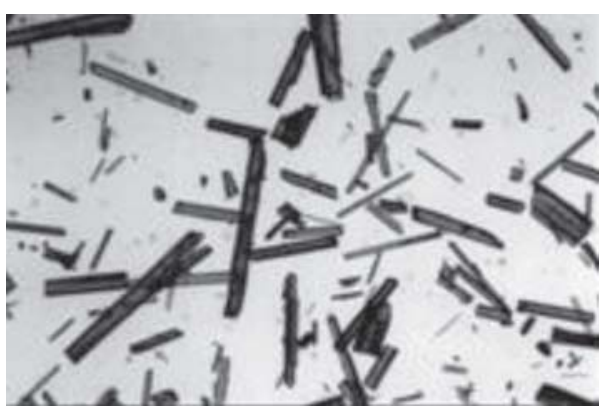

Figura 1. Microscopia óptica dos cristais de $\beta$-lapachona na objetiva $4 x$

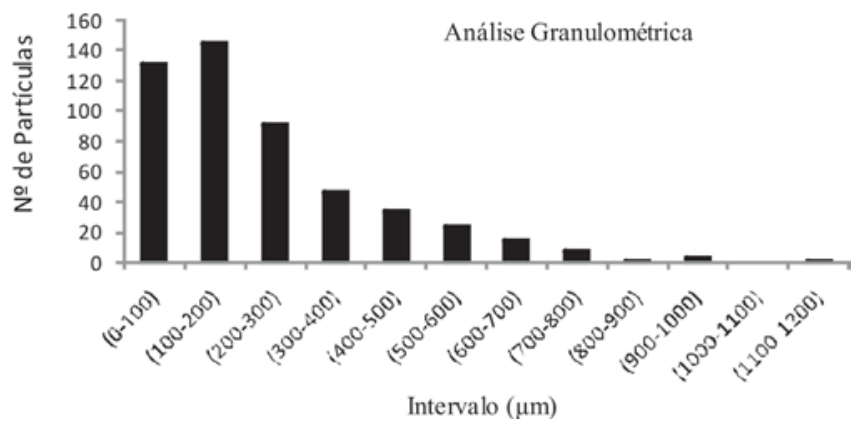

Figura 2. Gráfico da análise granulométrica

A umidade residual encontrada no Karl Fischer foi de 0,4247\%, enquanto que a encontrada pela perda por dessecação foi de $0,36 \%$. Esses resultados indicam que a $\beta$-lapachona possui uma boa proteção contra alterações que possam ser provenientes de uma higroscopicidade elevada, como é o caso de hidrólises.

$\mathrm{O}$ teste de metais pesados foi realizado seguindo os métodos gerais da Farmacopéia Brasileira IV, estando de acordo com a especificação colorimétrica ${ }^{14}$.

O doseamento por CLAE comprovou mais uma vez a pureza da substância, obtendo-se um cromatograma com apenas o pico característico da $\beta$-lapachona, com um teor de $100,77 \%$ e tempo de retenção de 6,9 min.

A curva de DSC mostrou um pico endotérmico característico de fusão em $155,46{ }^{\circ} \mathrm{C}$, com um calor de fusão de $91,01 \mathrm{~J} / \mathrm{g}$ e pureza de $99,67 \%$, evidenciando uma boa qualidade do processo de purificação da droga. Houve duas etapas de perda de massa detectadas pela curva da TG, a primeira com perda percentual de 70,6\% e a segunda com $26,8 \%$. Em seguida, ocorreu um processo de decomposição iniciado a $191{ }^{\circ} \mathrm{C}$ e finalizado em $250^{\circ} \mathrm{C}$, confirmado pelo DSC-fotovisual. A faixa de fusão da droga também foi confirmada pelo DSC-fotovisual nas temperaturas de 154,0 a $156,0{ }^{\circ} \mathrm{C}$, estando de acordo com a literatura e com a análise realizada em fusiômetro que apresentou valores de 154,5 a $155,4{ }^{\circ} \mathrm{C}{ }^{13}$.

As análises da $\beta$-lapachona por espectroscopia de infravermelho (IVTF), de ressonância magnética nuclear protônica $\left(\mathrm{RMN}{ }^{1} \mathrm{H}\right) \mathrm{e}$ de carbono $13\left(\mathrm{RMN}{ }^{13} \mathrm{H}\right)$ e espectrometria de massa (EM) e ultravioleta confirmaram a pureza e estrutura do composto através de seus picos, absorções e fragmentações características ${ }^{12,13}$.

$\mathrm{O}$ espectro de IVTF da amostra de $\beta$-lapachona apresentou banda de absorção característica de deformação axial de C-H aromático e alifático na região de 2977 e $2909 \mathrm{~cm}^{-1}$, respectivamente. Verificou-se a presença de harmônicas de combinação entre 2000 e 1800 $\mathrm{cm}^{-1} \mathrm{e}$ de banda em 1591 referente à deformação axial $\mathrm{C}=\mathrm{C}$ do anel. Podem ainda ser destacadas duas bandas na região de 1313,7 e $1117,0 \mathrm{~cm}^{-1}$ correspondentes às respectivas deformações axial assimétrica e simétrica de C-O-C. Foi observado também o sinal na região de $1694 \mathrm{~cm}^{-1}$ atribuído à banda de deformação axial de 
$\mathrm{C}=\mathrm{O}$; esta banda de absorção situada numa freqüência menor pode ser explicada pela forte influência do ângulo de ligação do anel que contém a carbonila. No caso da $\beta$-lapachona o ângulo é menor que 120 graus $^{12,13}$.

A partir da análise de $\mathrm{RMN}{ }^{1} \mathrm{H}$ da amostra foi observado um singleto em 1,448 ppm corresponde aos dois grupos metilênicos. Os prótons dos dois grupos metilênicos do éster cíclico são encontrados na forma de tripleto em 1,84 e 2,554 ppm, respectivamente. Os prótons referentes ao anel aromático são encontrados como duplo dupleto em 8,034 ppm (mais desblindado pela proximidade com a carbonila), 7,988; 7,63 e 7,488 ppm.

$\mathrm{O}$ espectro de massa da amostra de $\beta$-lapachona analisado revela o pico do íon molecular $\left(\mathrm{M}^{++1}\right)$ em m/z $242 \mathrm{~g} / \mathrm{mol}$, com $14 \%$ de abundância relativa. O pico de base em $\mathrm{m} / \mathrm{z} 159$ refere-se a fragmento iônico proveniente da abertura e rearranjo do ciclo com perda da parte alifática e de $\mathrm{C}=\mathrm{O}$.

A varredura espectrofotométrica por absorção ultravioleta mostrou um espectro idêntico ao obtido na literatura, com pico de absorção máxima em $256,00 \mathrm{~nm}^{15}$.

A $\beta$-lapachona demonstrou uma capacidade de compactação de $6 \mathrm{~mL}$, com densidade aparente e de compactação de 0,54 e 0,57 $\mathrm{g} / \mathrm{mL}$, respectivamente. Em relação à determinação do Índice de Carr e do Índice de Hausner os valores obtidos foram $\mathrm{I}_{\mathrm{CARR}}=5,26 \%$ e $\mathrm{I}_{\text {HAUSNER }}=1,06$. $\mathrm{O}$ resultado encontrado para a tangente entre altura e raio formado pelo escoamento do pó formou um ângulo de $28,73^{\circ}$ com um tempo de escoamento de 7,66 s. Estes valores indicam que apesar da forma cristalina, a $\beta$-lapachona apresenta propriedades de fluxo e compactação adequadas para desenvolvimento farmacotécnico.

\section{Estudo de estabilidade dos cristais}

Foi verificada uma instabilidade dos cristais expostos à luz fluorescente com mudança da cor de laranja para vinho logo com 30 dias de estudo. Essa coloração se intensificou com o passar do tempo. Ao atingir 180 dias de estudo foram realizadas análises de $\mathrm{RMN}{ }^{1} \mathrm{H}$, RMN ${ }^{13} \mathrm{C}$ e IVTF, onde ficou comprovado que havia ocorrido apenas uma alteração física de cor, sem alterações químicas detectáveis pelos métodos utilizados. Os cristais submetidos às outras condições de armazenamento não sofreram alterações visíveis.

Microscopicamente não houve diferença entre a morfologia dos cristais nas condições de armazenamento do estudo realizado nos tempos avaliados.

Através da análise de CCD foi possível perceber que em todas as condições estudadas não houve degradação das amostras em qualquer outra substância capaz de ser identificada através do sistema utilizado, apresentando somente uma mancha com fluorescência laranja característica da $\beta$-lapachona, com Rf de 0,25.

As análises realizadas por CLAE demonstraram que não houve diferenças significativas entre as condições de armazenamento e os tempos avaliados, quando tratadas estatisticamente por análise de variância two-way, pois o $\mathrm{F}$ calculado foi menor que o $\mathrm{F}$ crítico com um intervalo de confiança de $95 \%$ (Tabelas 1 e 2). Não houve diferença entre os cromatogramas das análises por CLAE, apresentando apenas o pico característico da $\beta$-lapachona como demonstrado no doseamento.

\section{CONCLUSÃO}

Os resultados obtidos mostram que o método de purificação utilizado é satisfatório para a obtenção de uma matéria-prima com
Tabela 1. Resultados do estudo de estabilidade dos cristais de $\beta$ lapachona em diferentes condições de armazenamento

\begin{tabular}{|c|c|c|c|c|c|}
\hline $\begin{array}{l}\text { Tempo/ } \\
\text { Condições }\end{array}$ & $\begin{array}{c}\text { Temp. } \\
\text { Ambiente } \\
(\mu \mathrm{g} / \mathrm{mL})\end{array}$ & $\begin{array}{r}\text { Dessecado } \\
(\mu \mathrm{g} / \mathrm{mL})\end{array}$ & efrigerado & $\begin{array}{l}\text { r Câmara } \\
\text { Climática } \\
(\mu \mathrm{g} / \mathrm{mL})\end{array}$ & $\begin{array}{c}\text { Exposto } \\
\text { à Luz } \\
(\mu \mathrm{g} / \mathrm{mL})\end{array}$ \\
\hline T0 & 5,003 & 5,091 & 5,039 & 5,086 & 5,031 \\
\hline T30 & 5,045 & 5,056 & 5,046 & 5,060 & 5,042 \\
\hline T60 & 5,026 & 5,046 & 5,088 & 5,075 & 5,074 \\
\hline T90 & 5,070 & 5,065 & 5,045 & 5,021 & 5,049 \\
\hline T180 & 5,022 & 5,060 & 4,988 & 4,988 & 5,034 \\
\hline
\end{tabular}

Tabela 2. Tratamento estatístico por análise de variância two-way do estudo de estabilidade dos cristais de $\beta$-lapachona

\begin{tabular}{lccc}
\hline Fonte de Variação & MQ & F calculado & F crítico \\
\hline Tempo & 0,001327 & 1,18837 & 3,007 \\
Condições & 0,002496 & 0,8857 & 3,007 \\
Residual & 0,000705 & & \\
\hline
\end{tabular}

alta qualidade de pureza, comprovada através da $\mathrm{CCD}$, que possibilita a separação do isômero $\alpha$-lapachona, um contaminante para a amostra em estudo. Dependendo da concentração de contaminação torna-se necessária a repetição do processo para a obtenção de uma matéria-prima pura. O sistema eluente utilizado possibilitou a separação das duas substâncias, sendo possível a identificação da $\alpha$-lapachona como contaminante. A matéria-prima purificada apresentou-se com padrões de qualidade para ser utilizada no processo de obtenção de uma forma farmacêutica. $\mathrm{O}$ estudo de estabilidade dos cristais de $\beta$-lapachona mostrou que não houve diferença entre as condições avaliadas, com exceção da amostra submetida à exposição à luz, que causou uma instabilidade física de coloração, devendo-se assim recomendar manter o produto sempre armazenado em frasco âmbar para evitar este desvio de qualidade.

\section{REFERENNCIAS}

1. Santana, C. F.; Gonçalves de Lima, O.; D’Albuquerque, I. L.; Lacerda, A. L.; Martins, D. G.; Rev. do Inst. de Antibiot. 1968, 8, 89.

2. Schuerch, A. R.; Wehrli, W.; Eur. J. Biochem. 1978, 84, 197.

3. Di Gianni, P.; Franco, M.; Bustuoabad, O. D.; Ruggiero, R.; Bol. A. N. de Medicina 1997, 75, 145.

4. Falkenberg, M. B. Em Farmacognosia: da Planta ao Medicamento; Simões, C. M. O., ed.; Eds. das Universidades UFRGS e UFSC: Porto Alegre/ Florianópolis, 2000, cap. 25.

5. Li, C. J.; Wang, C.; Pardee, A. B.; Cancer Res. 1995, 55, 3712.

6. Don, M. J.; Chang, Y. H.; Chen, K. K.; Ho, L. K.; Chau Y. P.; Mol. Pharmacol. 2001, 59, 784.

7. Boothman, D. A.; Trask, D. K.; Pardee, A. B.; Cancer Res. 1989, 49, 605

8. Phanchon, S. M.; Wuerzberger, S.; Frydman, B.; Witiak, D. T.; Hutson P.; Church, D. R.; Wilding, G.; Boothman, D. A.; Cancer Res. 1995, 55, 3706.

9. Prista, L. N.; Alves, A. C.; Morgado, R.; Tecnologia Farmacêutica, $5^{\mathrm{a}}$ ed.; Calouste Gulbenkian: Porto, 1995, vol. 1.

10. Wells, J. I.; Pharmaceutical Preformulation: The Physicochemical Properties of Drug Substances, John Wiley \& Sons: New York, 1988.

11. ANVISA, Agência Nacional de Vigilância Sanitária, Resolução 560, de 2 de abril de 2002, Guia para Realização de Estudos de Estabilidade, Diário Oficial da União, Brasília, Brasil, 03/04/2002.

12. Gonçalves de Lima, O.; D’Albuquerque, I. L.; Gonçalves de Lima, C.; Maia, M. H. D.; Rev. do Inst. de Antibiot. 1962, 4, 3.

13. D'Albuquerque, I. L.; Maciel, M. C. C.; Schuler, A. R. P.; Araujo, M. C. M.; Maciel, G. M.; Cavalcanti, M. S. B.; Martins, D. G.; Lacerda, A. L.; Rev. do Inst. de Antibiot. 1972, 12, 31.

14. Farmacopéia Brasileira, $4^{\mathrm{a}}$ ed.; Atheneu: São Paulo, 1988.

15. D'Albuquerque, I. L.; Rev. do Inst. de Antibiot. 1968, 8, 73. 